\title{
The Dynamics of Interactivity Modelling for e-Learning
}

\author{
Chima Adiele* \\ Department of Computing and Information Systems \\ Trinity Western University, Langley, BC, Canada \\ E-mail: chima.adiele@twu.ca
}

\section{Ezeamaka D. Nwanze}

Department of Computer Science University of Benin, Benin City, Nigeria

E-mail: dnwanze2000@yahoo.com

*Corresponding author

\begin{abstract}
Advances in information and communication technologies have given impetus to e-learning as choice educational environment for million of learners. E-learning involves the use of Internet technology to provide education where the instructor and students operate without geographical boundaries. Despite positive strides made in e-learning, the drop out rate of students remains high. Most educators argue that interactivity of learners is central to the success of e-learning initiatives. Accordingly, we present an interactivity model to dynamically measure interactivity in the context of elearning. The model leverages a common term vocabulary and Chebyshev's inequalities to objectively measure the contributions of participants in a group work. We evaluate the performance of our model using extensive simulation studies.
\end{abstract}

Keywords: E-learning, interactivity model, Web community and simulation.

Biographical notes: Chima Adiele received his Ph.D. in Computer Science from the University of Manitoba, Canada. Currently, Adiele is the Director of Policy, Planning \& Research (and Associate faculty) at Trinity Western University, Langley, BC, Canada. Adiele served as Faculty at the University of Lethbridge and Research Associate at the University of Manitoba, all in Canada He was a faculty and Coordinator of Diploma Programs at the University of Port Harcourt, Nigeria. Adiele's research essentially involves data manipulation and interpretation, including Web data integration, Internet computing systems and e-commerce. He has published his research work in refereed journals and conferences, and has also reviewed articles for reputable journals, including IEEE Internet Computing, IEEE Computer and IEEE Transactions on Knowledge and Data Engineering. Adiele is an active member of the IEEE and ACM.

Ezeamaka David Nwanze had his degrees from the University of Benin, Nigeria where he now serves as a senior faculty member. Nwanze has many years of teaching, research and industry experience. Nwanze's research focuses on the application of fuzzy logic in problem solving and the use of computers in education. Nwanze has published his research work in many refereed journals and conferences. 


\section{Introduction}

Advances in information and communication technologies provide great opportunities for creating virtual platforms where learners and instructors can interact in the framework of distance education. These opportunities are feasible because of the seamless global access that the Internet provides, and the user-friendly graphical interfaces that the Web supports. Avgeriou et al. (2003) observe that many software environments take advantage of the client-server communication on the Internet to support e-learning. E-learning involves the use of Internet technology to provide education where the instructor and students are partially or completely geographically dispersed. E-learning is also known as distance education, online learning, virtual classes, interactive learning and Web-based education in the literature.

People around the world are finding it necessary to constantly update their skills and knowledge in the current global economy, and this need is helping to fuel changes in education. These changes have given impetus to e-learning as choice educational environment for millions of learners. Castro et al. (2001) define a virtual campus as the group of functions which make interaction possible among the groups of people comprising a university (students, faculty, and management personnel) without requiring that they coincide in space or time. Beller and Or (1998) observe that the main advantages of distance education are availability, reduced cost, flexibility and integration. In the e-learning environment, students are capable of taking their courses from the comfort of their homes, often at their own pace, without necessarily disrupting their family lives.

Despite strides made in e-learning, many educators feel that the dropout rate is becoming increasingly unacceptable and point to interactivity as key to overcoming the problem (Elvheim, 2002; Khalifa \& Lam, 2002). Rafaeli (1988) defined Interactivity as "an expression of the extent that in a given series of communication exchanges, any third (or later) transmission (or message) is related to the degree to which previous exchanges referred to even earlier transmissions".

The main thrust of this paper is to dynamically measure interactivity of learners. To achieve the envisioned objective, we define the concept of interactivity in the context of e-learning, and hence, discuss the interactivity life cycle. We design an interactivity model that measures the interactivity of learners, and indeed, the interactivity level of groups of learners dynamically. We leverage a common term vocabulary to automatically filter irrelevant messages and promote interactivity. The model also uses Chebyshev's inequalities to classify members. We present simulation studies to evaluate the performance of our model.

The contributions of this paper include: Our interactivity model measures the interactivity of learners and the interactivity level of a group of learners. We leverage Chebyshev's inequality to classify members into four distinct groups. This classification is necessary as it provides a benchmark to reward learners. There is a lack of good tools to dynamically evaluate the participation of distance education students in group work (Hack \& Tarouco, 2000). Rewarding learners appropriately in group activities will elicit participation and increase interactivity (Lee, Cheung, \& Chen, 2005). This model provides a framework that instructors can leverage to more objectively reward learners in a group work.

The remaining part of this paper is structured as follows. In the next section, we discuss the e-learning interactivity life cycle, and present our model in the section follows immediately. In the next three sections, we examine some application areas of the 
interactivity model and present simulation results to evaluate the performance of our model. Finally, we review related works and draw reasonable conclusions.

\section{The Interactivity Life-Cycle}

Members of different Web communities (WCs) participate in different activities that generate messages and relate generated messages to existing ones. The activity that a member can participate in is a function of the category of WC the member belongs to. Fiore et al. (2002) identified a broad activity set for measuring interactivity. The set of activities include, authors, repliers, initiators, returning authors, posts, replies, thread starts (initial turns which received replies), barren posts (initial turns which received no replies), cross-posts, and cross-post targets (distinct groups with which this one shared messages).

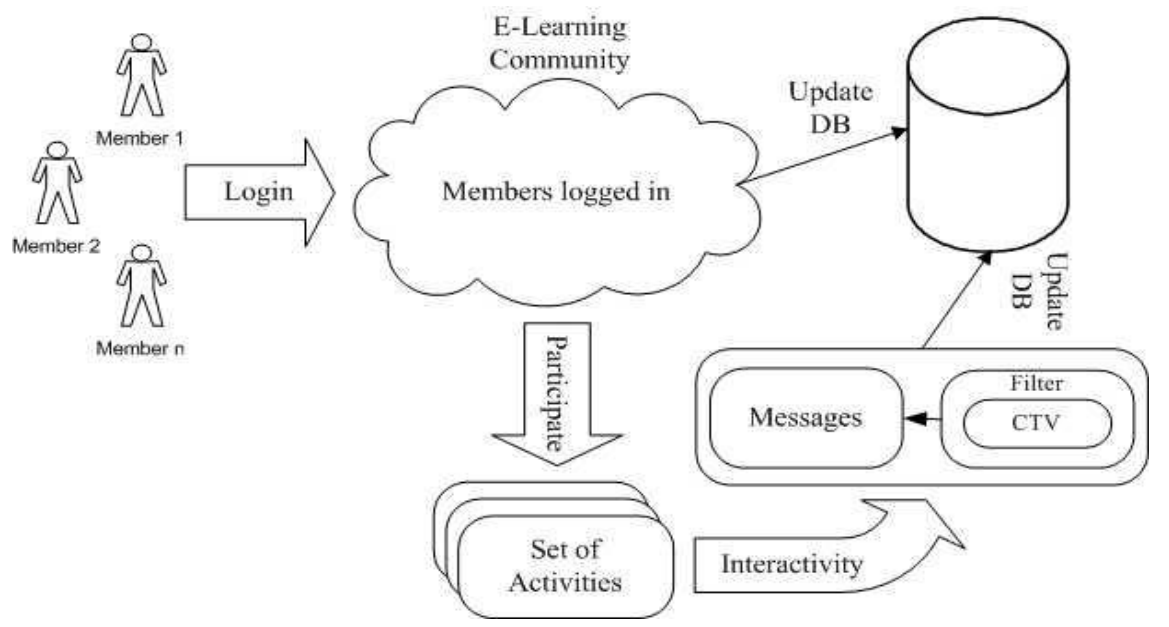

Figure 1. Interactivity Life-Cycle of an e-Learning Community

Figure 1 shows the interactions of members in a Web community. The system authenticates every member. This is important since the system requires that a user's actions be tracked. Hence, it is necessary to know who the user is. When a member logs into the system, the system automatically assigns a time stamp and extracts the member's user id to update the database. The system then monitors the activities that members will be involved in. A member can participate in several activities that may result in some measures of interactions. For example, a member can post a message or respond to messages posted. The system determines the level of interactivity and updates the database. The average time spent in the community and the set of activities a member can participate in varies from community to community.

A common problem in most WCs is the issue of posting irrelevant messages that have nothing to do with the subject of discussion, which sometimes may be offensive to some members of the community. To address this issue, some communities moderate messages posted. For example, Whittaker et al. (1998) argue for the necessity of knowledgeable moderators based on large scale empirical testing in the context of Usenet groups. Manually moderating messages, however, is fraught with problems. Such moderation is done by members with long experience in the community, and thus, their efforts increase common ground (the sense of commonality and understanding) among 
participants. In particular, the time taken to train such moderators is indeed enormous. The time and effort required to moderate a group is substantial, and grows rapidly with the size of the group. Obviously, not all communities, especially newer, smaller communities, have members willing to take on such a burden. In addition, such manual moderations are likely to be influenced by external factors, such as religion and politics (Ozturk \& Mutlu, 2005).

No doubt, manually moderating messages in large communities can be time consuming, labour intensive, and error prone. Therefore, there is a need to automate the process of filtering messages that are posted in a given community. We leverage a Common-Term Vocabulary (CTV) to automatically filter messages before they are posted. A CTV is an ontology that contains primitive terms in a given domain and does not prescribe any structure for its designers (Adiele \& Ehikioya, 2005b). The filter mechanism uses the CTV to filter messages before they are posted in the community. The filter mechanism is an accepting device that either accepts a message and it is posted, or rejects otherwise (Adiele \& Ehikioya, 2005a).

\section{The Interactivity Model for e-Learning}

To measure interactivity in a given e-learning community, we have to capture the set of activities, $A$ that are used to generate interactions in that community. Each activity; $a_{i} \in$ A, has a corresponding weight, $w_{i}$, which relates how important activity, $a_{i}$ is to the idea of interactivity in the given community.

Let $a_{i}$ be the ith activity in a set of activities, $A$ and $w_{i}$ be the corresponding weight of $a_{i}$. Then, we define interactivity in a given community as:

$$
\frac{\sum_{i=0}^{n}\left(w_{i}+a_{i}\right)}{n A}
$$

(where $n A$ is the number of activities in the set of activities, $A$ ).

We refer to the activities in equation (1) as primary activities. Some activities, however, are not interactive by themselves, but become interactive when an activity in the set of primary activities is performed by a user. For example, start post $(s P)$ is a way of posting messages in the community. A $s P$, however, is relevant to the extent that it relates to existing messages in the community. We assign the weight $\alpha$ to $s P$. The number of responses (Res) a $s P$ generates contributes to the value of the $s P$. Therefore, reply to a message counts in two ways: first, we assign the weight $\beta$ to the member who generates the reply $(r P)$; second, we assign the weight $\alpha$ to the member who initiates the $s P$ that is being replied to (Res). Observe Res is a dependant activity. Also, notice that the effective weight of $s P=\alpha+\alpha$.

These dependant activities are termed secondary activities. The performance of an activity $a_{i}$ may trigger zero or more secondary activities $a_{i j}$ as shown in Table 1. Observe from Table 1 that the participation of the primary activity $a_{2}$ triggers secondary activities $\mathrm{a}_{21}$ and $a_{22}$. 
Table 1. Activities and Corresponding Weights

\begin{tabular}{|l|c|c|}
\hline Activity & Weights & Trigger \\
\hline$a_{1}$ & $w_{1}$ & \\
\hline$a_{2}$ & $w_{2}$ & \\
\hline$a_{21}$ & $w_{21}$ & $a_{2}$ \\
\hline$a_{22}$ & $w_{22}$ & $a_{2}$ \\
\hline$a_{3}$ & $w_{3}$ & \\
\hline$\cdot$ & $\cdot$ & $\cdot$ \\
\hline$a_{n}$ & $w_{n}$ & \\
\hline
\end{tabular}

This implies that a secondary activity can contribute to the effective interactivity of a primary activity. Suppose a primary activity $a_{i}$ triggers secondary activities $a_{i j}$, (where $1<\mathrm{j} \leq \mathrm{m}$ ), the contributions of $a_{i j}$ to the interactivity of $a_{i}$ is given by:

$$
\sum_{j=0}^{m} w_{i j}\left(a_{i j}+a_{i}\right)
$$

Adding equations (1) and (2), we obtain:

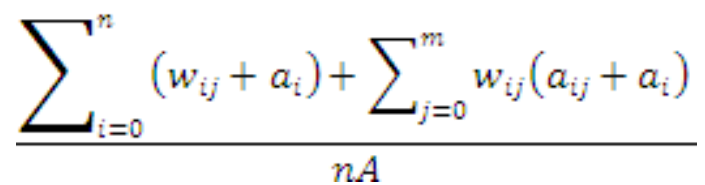

The square braces indicate that for primary activities that have no associated secondary activities, this part of the equation will evaluate to zero, and have no effect on the result.

Given a set of n members and an initial time window of $W_{i}^{o}=\left[e_{i}^{o}, l_{i}^{o}\right]$ associated to each member $i=1,2, \ldots, n$. Let $\hat{W}_{i}=\left[e_{i} ; l_{i}\right]$ be an alternative time window associated with each member $i=1,2, \ldots, n$. with $\hat{W}_{i} \geq W^{o}$. Let $s^{o}=l^{o}{ }_{i}-e^{o}{ }_{i}$ and $s_{i}=l_{i}-e_{i}$ be the width of the time windows in days with $s^{o}, s^{i} \in\{1,2, \ldots, l\}$ where $l$ is the maximum time width. Let $n_{j}$ be the number of members having time width equal to $j$.

To measure individual interactivity of a member, $m_{j}$ for a time window $\hat{W}_{i}$ (where $\hat{W}_{i}>\hat{W}_{i}{ }^{i}$ ), we compute the individual daily interactivity over the width, $S_{i}$ of $\hat{W}_{i}$. Accordingly, individual interactivity for $\hat{W}_{i}, I_{W I}$ is given by:

$$
I_{W I}=\sum_{S_{i}}\left\{\frac{\sum_{i=0}^{n} w_{i} a_{i}+\left[\sum_{j=0}^{m} w_{i j}\left(a_{i j}+a_{i}\right)\right]}{n A}\right\}
$$

Let $G S$ be the size of a Group with members $m_{j}$ (where $1<\mathrm{j}_{-}$GS) then, the Group interactivity $I_{W G}$ for a given time window Wi, width $\mathrm{Si}$ is given by: 


$$
I_{W G}=\sum_{G S} \sum_{S_{i}}\left\{\frac{\sum_{i=0}^{n} w_{i} a_{i}+\left[\sum_{j=0}^{m} w_{i j}\left(a_{i j}+a_{i}\right)\right]}{n A}\right\}
$$

Note that, given a WC with a set of $\mathrm{n}$ members, we can only compute interactivity for members with time windows of the same width, $S_{i}$. To compute the interactivity of a class $I_{W C}$, over a given time window $\hat{W}_{i}$, we sum the individual interactivity $I_{W I}$, over the size of the class.

Yap (2002) note that a community is made up of active and non active members, with majority of members in the non-active class. We measure members' contributions in the community by their level of interactivity. Accordingly, we classify members into two groups, namely active members and non-active members.

Definition 1: An active member is a member of a WC that contributes regularly to the development of the community.

Definition 2: A non-active member is a member of a WC that contributes sparingly to the development of the community.

Let AM and NM be sets of active and non-active members respectively. Let Mem be a power set of members, where:

$$
\begin{aligned}
& (A M \cup N M)=M e m \\
& (A M \cap N M)=0
\end{aligned}
$$

We model Members participation as a function of class of membership. Accordingly, the following inequalities hold:

$A N_{\text {пит }} \leq N M_{\text {пит }}$ (where "num" is the number of members)

$A N_{\text {cont }} \leq N M_{\text {cont }}$ (where "cont" is the contributions of members)

From Definition 1, we have that an active member contributes regularly to the interactivity level of the community. Therefore, an active member's contribution relative to the community interactivity level should be at least on the average. Combining Equation 4 with Definition 1, we obtain:

$$
I_{A M}=I_{W I}-\frac{\sum_{C S}\left(I_{W I}\right)}{C S} \geq 0
$$

From Definition 2, we have that a non-active member contributes sparingly to the interactivity level of the community. Therefore, a non-active member's contribution 
relative to the community interactivity level should be no more than the average interactivity level of the community. Combining Equation 4 with Definition 2, we obtain:

$$
I_{N M}=I_{W I}-\frac{\sum_{C S}\left(I_{W I}\right)}{C S}<0
$$

Equations (10) and (11) are the interactivity levels of active and non-active members respectively. Butler (2001) notes that an overwhelming majority of users are passive, while only $15 \%$ of users are leaders. This small percentage of members (leaders) in a WC is responsible for the majority of contributions (Butler, 2001). These leaders are active members that make substantial contributions to the interactivity level of the community. On the contrary, social loafers are non-active members that make no contributions at all to the interactivity level of the community. Let $L M$ and $S L$ be sets of leaders and social loafers respectively. We have that,

$$
L M \subset A M \wedge S L \subset N M
$$

The issue now is to determine members $l m$ and $s l$, such that $l m \in L M$ and $s l \in$ $S L$. We derive threshold bounds $\Phi$ and $\Theta$ for accumulated decisions using Chebyshev's inequality based on members' interactivity level over a given time window. Chebyshev's rule states that, for any number $k$ greater than 1 , at least $\left(1-1 / \mathrm{k}^{2}\right)$ of the measurements will fall within $\mathrm{k}$ standard deviations of the mean, i.e., within the interval $\left(x^{\prime}-k s, x^{\prime}+k s\right)$ for samples.

Let Max represent the maximum individual interactivity in a community and $\Phi=$ $x^{\prime}+2 s$. Let Min represent the minimum (zero) individual interactivity in a community and $\Theta=x^{\prime}-2 s$. We derive the following equations to determine leaders and social loafers in a community based on the community's interactivity level.

$$
\begin{aligned}
& l m \in L M \Longleftrightarrow \Phi \leq I_{W I} \leq \text { Max } \begin{cases}\text { leader : } & \text { if } s P \neq 0 \text { or } r P \neq 0 \\
A M: & \text { otherwise. }\end{cases} \\
& s l \in S L \Longleftrightarrow M i n \leq I_{W I}<\Theta \quad \begin{cases}\text { loafer: } & \text { if } s P=0 \text { and } r P=0 \\
N M: & \text { otherwise. }\end{cases}
\end{aligned}
$$

A member is a leader if the member's interactivity relative to the community's interactivity level for a given time window is greater or equal to the lower bound threshold, $\Phi$. In addition, this member must satisfy conditions in Equation 13. Similarly, a member is social loafer if the member's interactivity relative to the community's interactivity level for a given time window is strictly less than the upper bound threshold, $\Theta$. In addition, this member must satisfy conditions in Equation 14 .

\section{Dynamic Measures of Interactivity}

In this section, we describe the general simulation setup (including class setting, group participation, members' behavior patterns and interactivity computations) and explain our simulation results. Our model measures interactivity dynamically by capturing users' interactive activities (such as reading, posting and replying messages) in real time, and using the captured metrics to compute interactivity. 


\section{Activity Weights}

In every WC, an activity $a_{i} \in A$ has a measure of importance. This importance is captured by the weight $w_{i}$ assigned to the activity. The weight of an activity is assigned relative to the weight of a base activity. A base activity is a common activity in the community in which every member participates. Let $w_{1}, w_{2}, \ldots, w_{n}$ be weights of activities $a_{1}, a_{2}, \ldots, a_{n}$ respectively. Then,

$$
\sum_{i=1}^{n} w i=1
$$

Let the weight of the base activity be karma $(k)$ (a fair reward for a unit of the base activity). Let $w_{l k}, w_{2 k}, \ldots, w_{n k}$ represent the weights of activities $a_{1}, a_{2}, \ldots, a_{n}$ relative to $k$. This implies that:

$$
\begin{aligned}
& \sum_{i=1}^{n} w i(k)=1 \\
& k=\frac{1}{\sum_{i=1}^{n} w i}
\end{aligned}
$$

For example, if in a given community reading posts $(R)$ is the base activity in which every member participates. The weight of $R$ is karma $(k)$ since it is the base activity. Suppose there are three other activities in this community (say, $s P, \operatorname{Res}$, and $r P$ ), with relative weights of 4,3 , and 2 respectively. Then, from Equation (16), we have that: $4 k+3 k+2 k+k=1$; which implies that $k=0.1$. We can now determine the weights of the other activities from the weight of the base activity $(R=0.1)$.

\section{Simulation Environment}

We simulated our model using a discrete event generator, where members' participation and behaviors were modeled using a Poisson random process. To simplify our exposition, we restrict the number of activities to include:

1. Number of reads $(R)$ (the number of messages, message ${ }_{k}$ read by a member, $m_{j}$ during a given time window, $\left.W_{i}\right)$

2. Number of start posts $(s P)$ (the number of messages, message $e_{k}$ a member, $m_{j}$ initiates during a given time window, $W_{i}$ ),

3. Number of reply posts $(r P)$ (the number of replies to messages, message $e_{k}$ a member, $m_{j}$ generates during a given time window, $W_{i}$ ),

4. Number of replies (Res) a $s P$ generates during a given time window, $W_{i}$.

The weights $w_{j}$ of activities used in our simulation is randomly generated in the range $[1,10]$, such that $R$ is the base activity with weight, $k$. We constraint the random number generator for the weights such that the weight of $s P<\operatorname{Res}<r P$. From Equation (17), the weight of $R$ is given by $k=1 / \sum w_{i}$. For simplicity, we assume that the weights of these activities do not change for the duration of the simulation time.

Let $\operatorname{MaxSP} c$ be the maximum number of $s P$ for a given class of members, where class of members $\in\{L M, A M, N M\}$. Similarly, MaxRPc is the maximum number of $r P$ for a given class of members and MaxRc is the maximum number of $R$ for a given class 
of members. A member's daily $s P$ was generated randomly in the range [0, MaxSPc], such that for each class of members:

$$
\sum s P \leq \operatorname{MaxSPC}
$$

A member's daily $r P$ was generated randomly in the range of [0, MaxRPc], such that, for each class of members:

$$
\sum r P \leq \operatorname{MaxRPC}
$$

$$
\operatorname{MaxSPc}+\operatorname{MaxRPc}=t M c
$$

Notice that $t M c$ is the daily total messages for a given class of members, and $\sum t M c=t M$. A member's daily $R$ was generated randomly in the range of $[0, t M]$, such that, for each class of members, $0 \leq R \leq t M$.

\section{Class Interactivity}

Figure 2 shows the interactivity of students in a class in the context of a Web community. The class has 30 students and this figure is ordered according to individual interactivity score. Interactivity is seen to rise slightly for non-active members, slightly more for active members, and the final $15 \%$ of the graph, rises quite sharply indicating the substantial contribution leading members make.

Observe that the value of reads for most members was high, yet it had only slight effect on interactivity. Read on its own does not constitute interactivity.

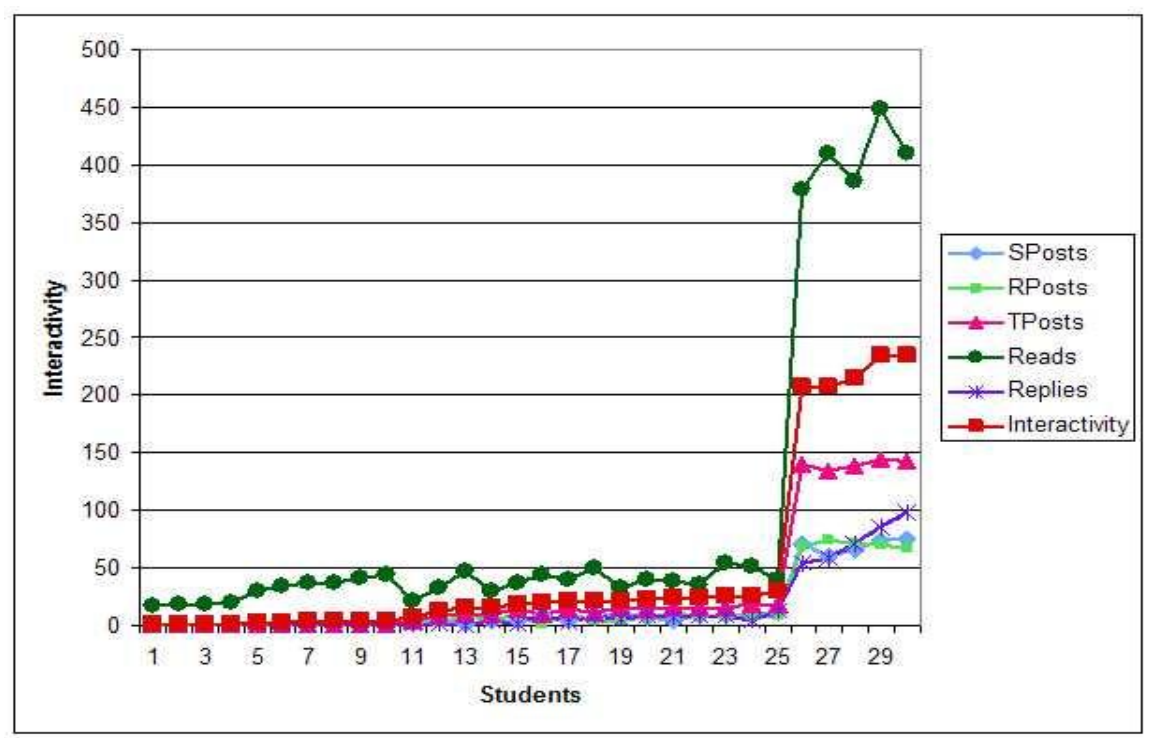

Figure 2. Interactivity of Members of a Class 
We leverage Chebyshev's inequality to classify members into groups as discussed in Equations (11) to (14). This classification provides an objective measure for instructors to reward students in group work according to their level of participation. We compute mean interactivity score $x$, (from Figure 2) for the class to be 47.7395837, while standard deviation s is 78.7417138. Therefore, $\Phi$ is 205.223011. Observe from Table 2 that members 01 to 11 are social loafers because they neither posted nor replied to messages. In addition, their interactivity is less than $x$ '. On the contrary, members 27 to 30 are leading members because they have contributed substantially, with their interactivity greater or equal to $\Phi$. Leading members represent $13.3 \%$ of the class, while social loafers represent $37.7 \%$ of the class. Overall, active members represent only $23.3 \%$ of the class, while the balance of $76.7 \%$ are non-active members.

\section{Group Interactivity}

Group work in normal classroom environment is a tool used by instructors to have students share ideals and develop the spirit of team work. One of the common problems of group work is the issue of under participation of members in a group. It is, therefore, necessary to reward members' contributions in a group work accordingly. Our model is able to give the instructor a number of important measures of interactivity when evaluating group work in an e-learning environment. The first of these is a comparison of group members. In each group, different people take on different roles, including those who fail to contribute entirely.

Table 2. Classification of Members of a Class

\begin{tabular}{|c|c|c|}
\hline Class & Members & Interactivity Level \\
\hline SL & $01-11$ & $s P=0$ and $r P=0$ \\
\hline NM & $12-23$ & $I_{W I}<\bar{x}$ and $(s P \neq 0$ or $r P \neq 0)$ \\
\hline AM & $24-26$ & $\Phi>I_{W I} \geq \bar{x}$ \\
\hline LM & $27-30$ & $I_{W I} \geq \Phi$ \\
\hline
\end{tabular}

Table 3. Interactivity of Members of Group-A

\begin{tabular}{|c|c|c|c|c|}
\hline Students & Student1 & Student2 & Student3 & Student4 \\
\hline Start Posts & 9 & 10 & 11 & 66 \\
\hline Reply Posts & 7 & 8 & 8 & 72 \\
\hline Total Posts & 16 & 18 & 19 & 138 \\
\hline Reads & 55 & 52 & 40 & 386 \\
\hline Replies & 8 & 5 & 13 & 72 \\
\hline Interactivity & 5.125 & 5.145833 & 5.916667 & 42.91667 \\
\hline
\end{tabular}




\section{Comparing Individuals}

Determining the contributions of members in a group can be very difficult for the instructor who is not privy to the group. The situation is even more difficult in an elearning environment where group members may be at different geographical locations and are unlikely to meet physically.

Table 3 shows the simulation of a group of four students. All the students in this simulation worked hard, with Student4 standing out from the others. It is obvious that Student 4 made far more contributions than the combined efforts of the other group members. Table 3 should guide the instructor to reward Student 4 more than the other group members, even though the common practice is to score the group as a whole. Table 4 shows simulated results for a group in which one student worked very hard, the other did not participate, and the other two students did the rest. Often times the instructor scores all the students in a group the same mark because of the difficulty in quantifying individual contributions in a group. No doubt, Student 1 did not contribute to the group work since all $\mathrm{s}(\mathrm{he})$ did was to read the contributions of others. We ran both simulations for 100 days and assumed overall daily contributions to the project to be 10 messages. With this information, the instructor makes a more informed decision about individual participation.

Table 4. Interactivity of Members of Group-B

\begin{tabular}{|c|c|c|c|c|}
\hline Students & Student1 & Student2 & Student3 & Student4 \\
\hline Start Posts & 0 & 4 & 5 & 76 \\
\hline Reply Posts & 0 & 6 & 9 & 67 \\
\hline Total Posts & 0 & 10 & 14 & 143 \\
\hline Reads & 20 & 30 & 37 & 410 \\
\hline Replies & 0 & 4 & 2 & 98 \\
\hline Interactivity & 0.416667 & 3.041667 & 3.791667 & 46.958333 \\
\hline
\end{tabular}

\section{The virtual Piazza}

A virtual piazza provides a framework for students to cross-fertilize ideas. Such ideas could be about courses they are enrolled in or other social activities. Ozturk and Mutlu (2005) investigate social interactivity in computer mediated communication in Turkey. The study found out that only approximately 50 percent of the students contributed messages that made it through the filters. It is very likely that external factors, such as religion and politics may have influenced contributions since such contributions are censored.

Participation in a virtual piazza may be voluntary or mandatory (Ozturk \& Mutlu, 2005). Voluntary participation is common in student socialization more than intellectual stimulation. Conversely, participation may be made mandatory, and part of the students' grades could even hinge on participation. The focus of this kind of forum would be more intellectual than social, though in either forum both might result.

Figure 3 shows the interactivity level of two classes. The first has mandatory participation (MP), and the second has voluntary participation (VP) in the forum. We examined their interactivity with varying thread lengths of messages. Observe that the class with MP had more interactivity than the class with VP. The class of MP reaches its peak of interactivity when thread length is 22 , while the class of VP reaches its peak of 
interactivity with a thread length of 52 . This shows that we have more active participants in the class of MP than the class of VP.

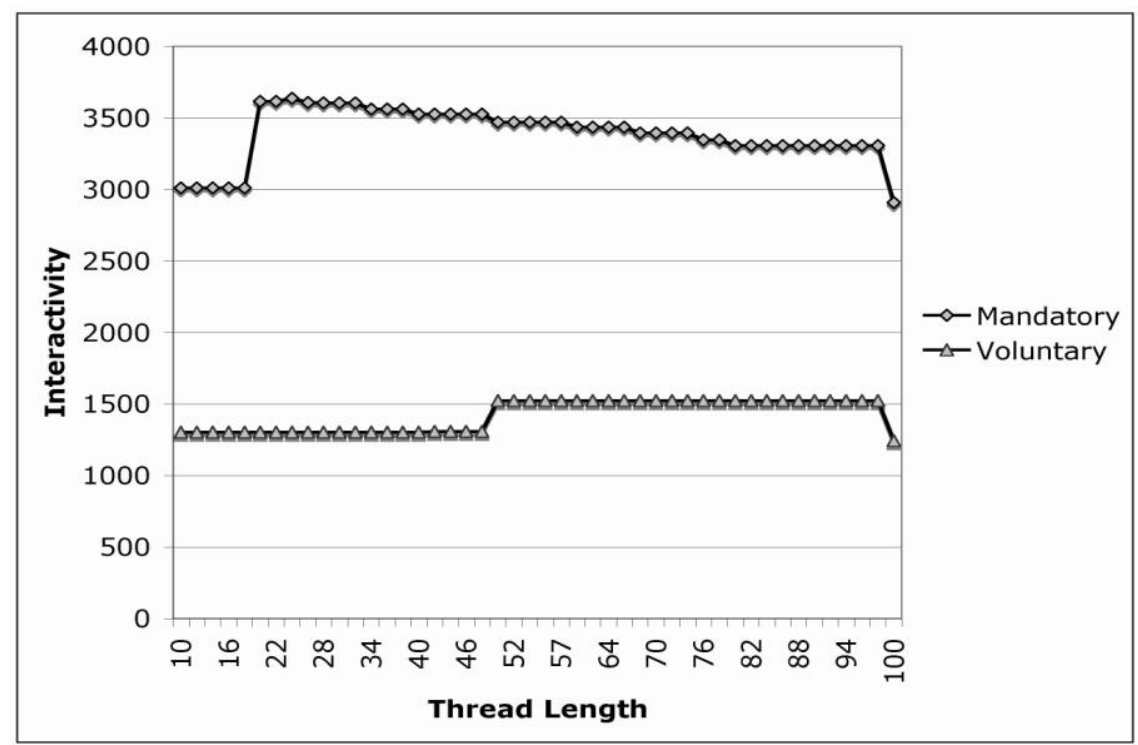

Figure 3. Measuring Interactivity with Varying Thread Lengths

\section{Related Work}

The expectations of the value in advancing information and communication technologies are well documented in the literature. Butler (2001) notes, however, that it is becoming increasingly obvious that providing electronic infrastructures could support social activity but does not guarantee its emergence. Lee et al. (2005) examine the role of intrinsic and extrinsic motivation in students' participation in e-learning. The paper notes that active participation and involvement of students is critical to the use of Internet technologies to support and improve learning in Internet-based learning medium. In education, Web communities have been deployed to complement traditional techniques such as lectures and tutorials (Dube, Bourhis, \& Jacob, 2006; Yang, Li, Tan, \& Teo, 2007). Zhang et al. (2006) argue that interactivity among learners in technology mediated education can improve the learning experience of participants and has positive effects on the student's performance.

Ozturk and Mutlu (2005) examine the importance of socialization in education. The authors argue that in distance education students need to improve their interactions and know others. These interactions enable students to associate for problem solving, and hence, effect changes in attitudes and behaviors of others. Such social interaction possibilities for distance learning will help decrease the isolation of those learners (Ozturk \& Mutlu, 2005). Saade and Huang (2009) investigated the contributions of online discussion forums in the context of e-learning by performing a macro-level analysis of performance and interaction. This analysis provided the authors the opportunity to better understand whether learning did occur and holistic behavior of students. 
Tarouca et al. (2000) present a learning environment developed to support group work and student assessment through Internet for distance education. The authors observe that it is necessary to develop applications to handle participants' contributions and to consolidate contributions by providing summaries of the discussion. Learning theories state that group learning has significant relevance and must be supported also in distance education. Instructors should be able to identify learners' participation in group activities and reward accordingly. Unfortunately, there is a lack of good tools to dynamically evaluate the participation of distance education students in group activities (Hack \& Tarouco, 2000). The model we present provides a framework that instructors can leverage to more objectively reward learners in a group work.

\section{Conclusions}

The increasing acceptance of e-learning as choice educational environment for millions of learners affirms the shift to a collaboration driven social networking system. Most learners take advantage of the opportunities provided by e-learning, such as availability, reduced cost, flexibility and integration to acquire formal education, while preserving their family or business lives. It has been observed that interactivity is key to the success of e-learning initiatives.

In this paper, we designed an interactivity model to dynamically measure the interactivity of students and the interactivity level of groups of students in the context of e-learning. In addition, the model uses Chebyshev's inequality to group members into distinct classes. We provided simulation results for interactivity at different levels of granularity to evaluate the performance of our model. For example, we examined the interactivity of students in a class showing how different students participate in class activities. We also discussed group interactivity and showed how an instructor could leverage this model to reward students in a group work more objectively. Providing appropriate rewards for learners in group activities will elicit participation and increase interactivity in the community.

The paper also discussed the concept of virtual piazza where participation in an elearning community could be voluntary or mandatory. Our simulation results showed that mandatory participation increases the interactivity level of a group, while voluntary participation does not produce a lot of active participants. Increased participation is necessary for sustained interactivity as it brings students together to share common goals and aspirations. The insight provided by the model could guide students, instructors, administrators and other stakeholders in the education industry. In the future, we intend to evaluate trust issues to ensure that the model satisfies both identity and behavioral trusts. 


\section{References}

1 Adiele, C., \& Ehikioya, S. A. (2005a). Algebraic signatures for scalable web data integration for electronic commerce transactions. Journal of Electronic Commerce Research, 6 (1), 56-74.

2 Adiele, C., \& Ehikioya, S. A. (2005b). Towards a formal data management strategy for a web-based community. Int. J. Web Based Communities, 1 (2), 226-242.

3 Avgeriou, P., Papasalouros, A., Retalis, S., \& Skordalakis, M. (2003). Towards a pattern language for learning management systems. Educational Technology \& Society, 6 (2), 11-24.

4 Beller, M., \& Or, E. (1998). The crossroads between lifelong learning and information technology - a challenge facing leading universities. Journal of Computer-Mediated Communication, 4 (2).

5 Butler, B. (2001). Membership size, communication activity and sustainability: a resource-based model of on-line social structures. Information Systems Research, 12 (4), 346-362.

6 Castro, M., López-Rey, A., Pérez-Molina, C. M., Colmenar, A., Mora, C. de, Yeves, F., Carpio, J., Peire, J., \& Daniel, J.S. (2001). Examples of distance learning projects in the European community. IEEE Transactions on Education, 44 (4), 406411.

7 Dube, L., Bourhis, L., \& Jacob, R. (2006). Towards a typology of virtual communities of practice. Interdisciplinary Journal of Information, Knowledge and Management, 1, 69-93.

8 Elvheim, M. (2002). Supporting group work in distance education. In The third international conference on extreme programming and flexible processes in software engineering (xp2002) (p. 204-205).

9 Fiore, A. T., Tiernan, S. L., \& Smith, M. A. (2002). Observed behavior and perceived value of authors in usenet newsgroups: bridging the gap. In Chi '02: Proceedings of the sigchi conference on human factors in computing systems ( $\mathrm{p}$. 323-330).

10 Hack, L., \& Tarouco, L. (2000). New tools for assessmentin distance education. In Proceedings of the society for information technology and teachereducation international conference (p. 241-244).

11 Khalifa, M., \& Lam, R. (2002). Web-based learning: Effects on learning process and outcome. IEEE Transactions on Education, 45 (2), 350-356.

12 Lee, M., Cheung, C., \& Chen, Z. (2005). Acceptance of internet-based learning medium: the role of extrinsic and intrinsic motivation. Information \& Management, 42 (8), 1095-1104.

13 Ozturk, M. C., \& Mutlu, M. E. (2005). An investigation of social interactivity in computer mediated communication. In 4th international symposium communication in the millennium. CIM.

15 Rafaeli, S. (1988). Interactivity: From new media to communication. In R. P. Hawkins, J. M. Wiemann, \& S. Pingree (Eds.), Sage annual review of communication research: Advancing communication science: Merging mass and interpersonal processes (Vol. 16, p. 110-134). Sage.

16 Saade, R. G., \& Huang, Q. (2009). Meaningful learning in discussion forums: Towards discourse analysis. Issues in Informing Science and Information Technology, 6 , 87-99. 
17 Tarouco, L., Geller, M., Vit, A., \& Hack, L. (2000). Supporting group learning and assessment through internet. In Proceedings of the society for information technology and teacher education international conference (p. 143-147).

18 Whittaker, S., Terveen, L., Hill, W., \& Cherny, L. (1998). The dynamics of mass interaction. In Cscw '98: Proceedings of the 1998 ACM conference on computer supported cooperative work (pp. 257\{264). New York, NY, USA: ACM Press.

19 Yang, X., Li, Y., Tan, C., \& Teo, H. (2007). Students' participation intention in an online discussion forum: Why is computer-mediated interaction attractive? Information \& Management, 44(5), 456-466.

20 Yap, A. Y. (2002). Enabling e-commerce growth through the social construction of a virtual community's culture. Journal of Electronic Commerce Research, 3 (4), 279-294.

21 Zhang, D., Zhou, L., Briggs, R., \& Nunamaker, J. (2006). Instructional video in elearning: Assessing the impact of interactive video on learning effectiveness. Information \& Management, 43 (1), 15-27. 